TITLE:

\title{
Estimates of the Transition Densities for Brownian Motion on Nested Fractals
}

$\operatorname{AUTHOR}(\mathrm{S}):$

KUMAGAI, TAKASHI

CITATION:

KUMAGAI, TAKASHI. Estimates of the Transition Densities for Brownian Motion on Nested Fractals. 数理解析研究所講究録 1992, 783: 27-45

ISSUE DATE:

1992-05

URL:

http://hdl.handle.net/2433/82551

RIGHT: 


\title{
Estimates of the Transition Densities for Brownian Motion on Nested Fractals.
}

\author{
TAKASHI KUMAGAI \\ Department of Mathematics, Osaka University \\ Toyonaka, Osaka 560, Japan
}

\section{$\S 0$ Introduction}

Aronson type estimates of the transition densities for Brownian motion are obtained in the case of the Sierpinski gasket by Barlow-Perkins [4] and in the case of the Sierpinski carpet (which is not a nested fractal) by Barlow-Bass [3]. In this paper, we will generalize them on nested fractals introduced by Lindstrøm [9], which is a class of finitely ramified fractals and contains Sierpinski gasket as a typical example.

The analysis of the Brownian motion on nested fractals has been studied by Lindstrøm [9] using nonstandard analysis and by Kusuoka [8] and Fukushima [5] using Dirichlet forms. But we construct the Brownian motion as the limit of a random walk by using the theory of multi-type branching processes. It is a generalization of the methods of Barlow-Perkins [4] which reduced the construction to the theory of branching processes. Our main theorem is as follows:

Let $p(t, x, y)$ be a continuous version of the transition densities of the Brownian motion $X_{t}$ with respect to the Hausdorff measure on the unbounded nested fractals $\tilde{F}$ which satisfies Assumption 2.2 (see $\S 2$ ). Then there exist positive constants $c_{1} \sim c_{4}$, such that

$c_{1} t^{-d_{s} / 2} \exp \left(-c_{2}\left(|x-y|^{d_{w}} / t\right)^{1 /\left(d_{J}-1\right)}\right) \leq p(t, x, y) \leq c_{3} t^{-d_{s} / 2} \exp \left(-c_{4}\left(|x-y|^{d_{w}} / t\right)^{1 /\left(d_{J}-1\right)}\right)$

for all $t>0, x, y, \in \tilde{F}$.

Here $d_{s}$ is a constant which expresses the asymptotic behavior of the eigenvalue of the corresponding generator $\Delta$, and $d_{w}$ is related to the diffusion constant.

I.e. $\sharp\{\lambda \mid \lambda$ is a eigenvalue of $-\Delta, \lambda \leq x\} \sim x^{d_{s} / 2}$ and $E\left(\left|X_{t}\right|\right) \sim t^{1 / d_{w}} . d_{J}$ is a constant related to the order of the shortest path in nested fractals (see $\S 3$ for details). In the case of Sierpinski gasket and carpet, $d_{J}=d_{w}$.

We will follow the way of [3] and [4]. Technically the main key point is to study the behavior of the probability distribution of the almost-sure limit random variable in the multi-type branching process.

The author would like to express his sincere gratitude to Professor Kusuoka. Without discussions with him, the author would not have solved the problems. 


\section{$\S 1$ Nested fractals.}

In this section, we will remember the definitions and geometrical properties of nested fractals. Although all the results are obtained by Lindstrøm [9], we will follow notations to Kusuoka [8].

Definition 1.1. Let $\alpha>1, D \in \mathbb{N}$. We say that $\psi: \mathbb{R}^{D} \rightarrow \mathbb{R}^{D}$ is an $\alpha$-similitude, if $|\psi(x)-\psi(y)|=\alpha^{-1}|x-y|$ for any $x, y \in \mathbb{R}^{D}$.

Let $\alpha>1$ and $\left\{\psi_{1}, \cdots, \psi_{N}\right\}$ be an $\alpha$-similitudes in $\mathbb{R}^{D}$. Then, there exits unique compact set $E$ which satisfies $E=\cup_{i=1}^{N} \psi_{i}(E)$ (c.f. Hutchinson [6]). We call this $E$ a self-similar fractal. In the following, we normalize $\operatorname{diam} E=1$.

Definition 1.2. Let $F$ be the set of fixed points of $\psi_{i}$ 's, $1 \leq i \leq N . x \in F$ is called an essential fixed point if there exist $i, j \in\{1, \cdots, N\}, i \neq j$ and $y \in F$ such that $\psi_{i}(x)=$ $\psi_{j}(y)$. We denote by $F^{(0)}$ the set of essential fixed points.

Notation 1.3 .

1) For $A \subset \mathbb{R}^{D}$ and $i_{1}, \cdots, i_{n} \in\{1, \cdots, N\}, A_{i_{1} \cdots i_{n}}$ denotes the set $\psi_{i_{1}}\left(\cdots \psi_{i_{n}}(A) \cdots\right)$.

2) Let $F^{(n)}=\cup_{i_{1}, \cdots, i_{n}=1}^{N} F_{i_{1} \cdots i_{n}}^{(0)}$ for each $n \geq 1$. Further, let $F^{(\infty)}=\cup_{n \in \mathbb{N}} F^{(n)}$. Then, its closure in $\mathbb{R}^{D}$. corresponds to the self-similar fractal $E=C l\left(F^{(\infty)}\right)$ (c.f. Hutchinson [6]). 3) For each $n \geq 0$, a set of the form $F_{i_{1} \cdots i_{n}}^{(0)}$ is called an $n$-cell, and a set of the form $E_{i_{1} \cdots i_{n}}$ is called an $n$-complex.

We will impose some assumptions on the family $\left\{\psi_{1}, \cdots, \psi_{N}\right\}$ to define nested fractals.

(A-0): (Open set condition) $\left\{\psi_{1}, \cdots, \psi_{N}\right\}$ satisfies the open set condition.

(A-1): (Connectivity) For any two 1-cells $C$ and $C^{\prime}$, there is a sequence $\left\{C_{i}: i=\right.$ $0, \cdots, n\}(n \in \mathbb{N})$ of 1 -cells such that $C_{0}=C, C_{n}=C^{\prime}$ and $C_{i-1} \cap C_{i} \neq \phi, i=1, \cdots n$.

(A-2): (Symmetry) For any $x, y \in \mathbb{R}^{D}$ with $x \neq y, H_{x y}$ denotes the hyperplane given by $H_{x y}=\left\{z \in \mathbb{R}^{D}:|z-x|=|z-y|\right\}$, and $U_{x y}$ denotes the reflection with respect to $H_{x y}$.

If $x, y \in F^{(0)}$ and $x \neq y$, then $U_{x y}$ maps $n$-cells to $n$-cells, and maps any $n$-cell which contains elements in both sides of $H_{x y}$ to itself for each $n \geq 0$.

(A-3): (Nesting) If $n \geq 1$ and if $\left(i_{1}, \cdots, i_{n}\right)$ and $\left(j_{1}, \cdots, j_{n}\right)$ are distinct elements of $\{1, \cdots, N\}^{n}$, then

$$
E_{i_{1} \cdots i_{n}} \cap E_{j_{1} \cdots j_{n}}=F_{i_{1} \cdots i_{n}}^{(0)} \cap F_{j_{1} \cdots j_{n}}^{(0)} \text {. }
$$

Definition 1.4. A self-similar fractal $E$ associated with $\alpha$-similitudes $\left\{\psi_{1}, \cdots, \psi_{N}\right\}$ is called a nested fractal if it satisfies the assumptions (A-0) $\sim(\mathrm{A}-3)$ and $\sharp F^{(0)} \geq 2$.

The following result is by Hutchinson [6]. 
THEOREM 1.5. The Hausdorff dimension $d_{f}$ of the nested fractal $E$ is $\frac{\log N}{\log \alpha}$.

\section{NOTATION 1.6 .}

Let $l_{1}, \cdots, l_{r}$ be such that $0<l_{1}<\cdots<l_{r}$ and $\left\{l_{1}, \cdots, l_{r}\right\}=\left\{|x-y|: x, y \in F^{(0)}, x \neq\right.$ $y\}$. For each $x \in F^{(m)}$, let $N_{m}^{i}(x) \in F^{(m)}$ be one of the $F^{(m)}$-neighbors of $x$ such that $\left|x-N_{m}^{i}(x)\right|=\alpha^{-m} l_{i}$ for $1 \leq i \leq r$. We omit $m$ when $m=0$. We call a path from $x$ to $N_{m}^{i}(x)$ a path of type $<i>, 1 \leq i \leq r$.

Finally, we list up geometrical properties of nested fractals obtained by Lindstrøm [9].

Proposition 1.7.

(1) If $x, y, x^{\prime}, y^{\prime} \in F^{(0)}$ and $|x-y|=\left|x^{\prime}-y^{\prime}\right|$, then there is a symmetry $U$ (i.e. reflection in $(A-2)$ ) such that $U(x)=x^{\prime}$ and $U(y)=y^{\prime}$.

(2) Any 1-cell contains at most one element of $F^{(0)}$.

(3) Let $x, y \in F^{(1)}$. Then there is a strict 1 -walk $s_{1}, \cdots, s_{n}$ (i.e. $s_{i}$ and $s_{i+1}$ are $F^{(1)}$ neighbors and $\left.\left|s_{i}-s_{i+1}\right|=\alpha^{-1} l_{1}, 1 \leq i \leq n-1\right)$ such that $s_{1}=x, s_{n}=y$ and $s_{k} \in$ $F^{(1)}-F^{(0)}, k=2, \cdots, n-1$.

\section{$\S 2$ Construction of the Brownian motion on nested fractals.}

In this section, we construct the Brownian motion on nested fractals. The proofs are almost the same as that of [7]. Thus we only remark necessary modifications in the proofs.

We fix one of $F^{(0)}$ and call it the origin. Define inductively $F_{n}=\alpha^{n} F^{(n)}(n \geq 0)$. (Here we denote $\lambda A=\{\lambda x: x \in A\}$.) Now we change the definition of $F^{(n)}$ as follows: $F^{(0)} \equiv \cup_{n=0}^{\infty} F_{n}$ and $F^{(n)} \equiv \alpha^{-n} F^{(0)}$ for $n \in \mathbb{Z}$. We denote $\tilde{E}=\mathrm{Cl}\left(\cup_{n \in \mathbb{Z}} F^{(n)}\right)$. Thus $\tilde{E}$ is a nested fractal which is extended to infinity. We will construct the Brownian motion on $\tilde{E}$.

First, we give some notations to explain our ideas exactly.

\section{NOTATION 2.1:}

1) For $x \in F^{(m)}$, let $\rho(x)=\sharp\{C: C$ is a $m$-cell containing $x\}$.

Also, for $x, y \in F^{(m)}, x \neq y$, let

$\tilde{\rho}(x, y)=\sharp\{C: C$ is a $m$-cell containing both of $x$ and $y\}$.

2) For the $\tilde{E}$-valued process $X(t), t \geq 0$, set

$T^{m}(X)=T_{0}^{m}(X)=\inf \left\{t \geq 0: X(t) \in F^{(m)}\right\}$,

$T_{i+1}^{m}(X)=\inf \left\{t>T_{i}^{m}(X): X(t) \in F^{(m)}-\left\{X\left(T_{i}^{m}(X)\right)\right\}\right\}, \quad i \geq 0$.

In the same way, set $T(A, X)=\inf \{t \geq 0: X(t) \in A\} \quad$ for $A \in E$.

Throughout this paper, we assume the following assumption on nested fractals. 
Assumption 2.2. There exists $k \in \mathbb{N}$ satisfying the following.

If $x, y \in E$ satisfy $|x-y| \leq \alpha^{-m}$, then there exist $x_{i_{1}}, x_{i_{2}}, \cdots, x_{i_{l}} \quad(l \leq k)$ such that $x_{i_{1}}=x, x_{i_{l}}=y, \quad x_{i_{2}}, \cdots, x_{i_{l-1}} \in F^{(m)}$ and $x_{i_{j}}, x_{i_{j+1}}$ join in the same $m$-complex for $1 \leq j \leq l-1$.

Let $\left\{Y_{r}\right\}_{r=0}^{\infty}$ be a random walk on $F^{(1)}$ starting at 0 with the following transition probabilities:

$$
P\left(Y_{r+1}=y \mid Y_{r}=x\right)=\rho(x)^{-1} \tilde{\rho}(x, y) p_{i} \equiv P(x, y) \quad \text { if }|x-y|=\alpha^{-1} l_{i}, 1 \leq i \leq r .
$$

Here, $\mathbf{p}=\left(p_{1}, \cdots, p_{r}\right)$ is the fixed point of [8] Theorem (3.10) which satisfies $p_{1}>\cdots>$ $p_{r}>0$ and $\sum_{i=1}^{r} m_{i} p_{i}=1$. [ $m_{i} \equiv \sharp\left\{y:|x-y|=l_{i}\right\}$ for $x \in F^{(0)} \cap E\left(m_{i}\right.$ is independent of the choice of $\left.x \in F^{(0)}\right)$.] Then, this random walk satisfies the following decimation property.

$\tilde{Y}(i) \equiv \alpha^{-1} Y\left(T_{i}^{0}(Y)\right), i \geq 0$ has the same distribution as $Y$.

In the following, we fix this transition probability and call this random walk as the decimation random walk. (Existence of the decimation random walk is proved by Lindstrøm [9] and uniqueness is proved in some special cases by Barlow [2].)

Notation 2.3:

1) Let $\eta_{i}$ be the number of times which $Y$ has passed paths of type $<i>$ before $T_{1}^{0}(Y)$, $1 \leq i \leq r$.

2) Let $n \geq m$. For a $F^{(n)}$-valued random walk $X_{n}$, let $T_{i}^{m<l>}\left(X_{n}\right)$ be the number of times which $X_{n}$ has passed $<l>$-type paths before the time $T_{i}^{m}\left(X_{n}\right)$, and set $W_{i}^{m<l>}\left(X_{n}\right)=T_{i}^{m<l>}\left(X_{n}\right)-T_{i-1}^{m<l>}\left(X_{n}\right)(i \geq 1)$.

These random variables are well defined because of Proposition 1.7 (1). For $x \in F^{(0)}$, define

$$
f^{l}\left(s_{1}, \cdots, s_{r}\right)=E^{x}\left(s_{1}^{\eta_{1}} \cdots s_{r}^{\eta_{r}} \mid Y\left(T_{1}^{0}(Y)\right)=N^{l}(x)\right)
$$

Remark that this $f_{l}$ is independent of the choice of $x \in F^{(0)}$. Further, this $f_{l}$ is a fractional function as it is the solution of a system of linear equations (c.f. [7]).

Let $\left\{X(n, x): x \in F^{(n)}\right\}$ be a family of decimation random walks which satisfies the following properties:

(1) $\{X(n, x): n \in \mathbb{Z}\}$ is a decimation random walk on $F^{(n)}$ starting at $x$.

(2) If $m \leq n$ and $x \in F^{(m)}$, then $X(m, x)(i)=X(n, x)\left(T_{i}^{m}(X(n, x))\right), \quad i \geq 0$.

(3) $X(n, x)(i)=X\left(n, X(n, x)\left(T^{j}(X(n, x))\right)\right)\left(i-T^{j}(X(n, x))\right)$ for $i \geq T^{j}(X(n, x)),-\infty \leq j \leq n, x \in F^{(n)}$.

(4) If $n, j \in \mathbb{Z}$ and $n \geq j$, then $\sigma\left\{X(n, y), y \in F^{(j)}\right\}$ and $\sigma\left\{X(n, x)\left(\cdot \wedge T^{j}(X(n, x))\right), x \in F^{(n)}\right\}$ are independent $\sigma$-fields.

We have the following key lemma. 
LEMMA 2.4 .

(a) If $x \in F^{(n)}, n \geq m$, then $\left\{\left(W_{i}^{m<l>}(X(n, x))\right)_{l=1}^{r}: i \in \mathbb{N}\right\}$ are i.i.d. random vectors whose common distribution does not depend on $x$. Hence, $\left\{W_{i}^{m}(X(n, x)): i \in \mathbb{N}\right\}$ are i.i.d. whose distribution does not depend on $x$.

(b) If $m \in \mathbb{Z}, i \in \mathbb{N}$, and $x \in F^{(m)}$ are fixed, then the $r$-dimensional process

$$
t \rightarrow \mathbf{Z}_{t}=\left(Z_{t}^{<l>}, 1 \leq l \leq r\right)
$$

where $\quad Z_{t}^{<l>}=W_{i}^{m<l>}(X(m+t, x)), \quad 1 \leq l \leq r, \quad t \in \mathbb{Z}_{+} \quad\left(\mathbb{Z}_{+} \equiv \mathbb{N} \cup\{0\}\right)$ is a multi $r$-type branching process. The $<l>$-type offspring distribution of the type $\langle k\rangle$ equals to the law of $\eta_{l}$ under the conditional probability $P\left(\cdot \mid Y\left(T_{1}^{0}(Y)\right)=N^{k}(0)\right)$ for $1 \leq l, k \leq r$. Note that the distribution of $Z_{t}^{<l>}$ does not depend on $m$ nor $i$.

Let $M$ be the $r \times r$-matrix such that

$$
M=\left(\frac{\partial f^{i}}{\partial s_{j}}(1, \cdots, 1)\right)=\left(E\left(\eta_{j} \mid Y\left(T_{1}^{0}(Y)\right)=N^{i}(0)\right)\right) .
$$

By Proposition $1.7(2),(3), M$ is a positive matrix. Let the largest eigenvalue of $M$ be $t_{E}$. If we let $\vec{v}=\left(m_{1} p_{1}, \cdots, m_{r} p_{r}\right)$, then $(\vec{v}, 1)=1$. Further,

$$
\vec{v} M=\left(E\left(\eta_{1}\right), \cdots, E\left(\eta_{r}\right)\right)
$$

and by the optional stopping theorem, the right hand side is a constant multiple of $\vec{v}$. Thus, by the Frobenius theory, $\vec{v}$ is an eigenvector for $t_{E}$. By Proposition $1.7(2), \sum_{i=1}^{r} E\left(\eta_{i}\right) \geq 2$. Hence we have $t_{E} \geq 2$.

For $n \in \mathbb{Z}$ and $x \in F^{(n)}$, let $X_{n}(x)\left(j \cdot t_{E}{ }^{-n}\right)=X(n, x)(j)$ and extend $X_{n}(x)(t)$ to $t \in[0, \infty)$ by an adequate interpolation in $\tilde{E}$ so that $X_{n}(x)(\cdot) \in C([0, \infty), \tilde{E})$.

Proposition 2.5. Let $m \in \mathbb{Z}$ and $x \in F^{(m)}$.

(a) For each $i \in \mathbb{N}, 1 \leq l \leq r, \quad W_{i}^{m<l>}\left(X_{n}(x)\right)$ converges a.s. and in $\mathbb{L}^{2}$ as $n \rightarrow \infty$ to $m_{l} p_{l} W_{i}^{m}(x)$, where $W_{i}^{m}(x)$ is a random variable which is strictly positive a.s.

(b) $\left\{W_{i}^{m}(x): i \in \mathbb{N}\right\}$ are i.i.d. random variables.

(c) $W_{i}^{m}(x)$ is equal in law to $W_{1}^{0}(0) \cdot t_{E}{ }^{-m}$.

If $\quad \phi_{l}(s)=E\left(e^{-s W_{1}^{0}(0)} \mid \mathbf{Z}_{0}=\mathbf{e}_{l}\right), \quad \operatorname{Re} s \geq 0,1 \leq l \leq r$,

where $\mathbf{e}_{l}$ is the unit vector whose $l$-th component is 1 , then $\phi_{l}$ satisfies

$$
\phi_{l}\left(t_{E} s\right)=f^{l}\left(\phi_{1}(s), \cdots, \phi_{r}(s)\right) \quad \text { for } \operatorname{Re} s \geq 0,1 \leq l \leq r .
$$

Proof: Just as [7], we apply the general theory of supercritical multi-type branching processes (c.f. Athreya-Ney [1]). We remark again that $t_{E} \geq 2, M>0, f_{l}$ is a fractional 
function and $f_{l}(0, \cdots, 0)=0$. It is easy to check the conditions required for the general theory if we use these facts.

We denote $T_{j}^{m}(x)=\sum_{i=1}^{j} W_{i}^{m}(x)$ for $x \in F^{(m)}$. Also we use $W$ to denote a random variable equal in law to $W_{1}^{0}(x)$.

Theorem 2.6. For each $x \in F^{(\infty)}, X_{n}(x)$ converges a.s. in $C([0, \infty), \tilde{E})$ as $n \rightarrow \infty$ to a process, $X(x)$. Moreover, for all $m \in \mathbb{Z}, j \in \mathbb{Z}_{+}$and $x \in F^{(m)}$,

$$
X(x)\left(T_{j}^{m}(x)\right)=X(m, x)(j) .
$$

Denote by $\mathbb{L}^{0}(C([0, \infty), \tilde{E}))$ the complete metric space formed of $C([0, \infty), \tilde{E})$-valued random vectors with the topology of convergence in probability. Then we have the following proposition in the same way as [7]. Remark that the Assumption 2.2 is necessary for the proof.

Proposition 2.7. The mapping

$$
X: F^{(\infty)} \rightarrow \mathbb{L}^{0}(C([0, \infty), \tilde{E}))
$$

is uniformly continuous on bounded subsets of $F^{(\infty)}$ and hence has a unique continuous extension to $\tilde{E}$, which we also denote by $X$.

Let $\Omega=C([0, \infty), \tilde{E}), P^{x}$ be the law of $X(x)$ on $\Omega$, and $\mathcal{F}$ be the Borel $\sigma$-field on $\Omega$. Then we have the following theorem.

ThEOREM 2.8. $\left(\Omega, \mathcal{F}, P^{x}\right)$ is a Feller diffusion process, that is, it is a continuous strong Markov process such that $P_{t}: C_{b}(\tilde{E}) \rightarrow C_{b}(\tilde{E})$. Here $C_{b}(A)$ is a set of continuous bounded functions on $A$.

Definition 2.9: We call this process as the Brownian motion on $\tilde{E}$.

Lemma 2.10. Let $A$ be an open subset of $\tilde{E}$ such that $\partial A$ is a finite subset of $F^{(\infty)}$. ( Remark that $\partial A$ is a topological boundary of $A$ by considering $A \subset \tilde{E}\left(\subset \mathbb{R}^{D}\right)$.)

(a) $T^{m}(x)=T^{m}(X(x))$ for all $m \in \mathbb{Z}$ and $x \in F^{(\infty)}$ a.s.

(b) $T_{i}^{m}(x)=T_{i}^{m}(X(x))$ for all $m \in \mathbb{Z}, i \in \mathbb{N}$ and $x \in F^{(m)}$ a.s.

Let $\mu$ be the $d_{f}$-Hausdorff measure on $\widetilde{E}$ such that $\mu(E)=1$. Also we define a probability measure $\mu_{n}$ on $F^{(n)}$ by 


$$
\mu_{n}(x)=\frac{\rho(x)}{N^{n} \sharp F^{(0)}} \quad \text { for } x \in F^{(n)} \text {. }
$$

Then, $\left\{\mu_{n}\right\}$ converges vaguely to $\mu$. I.e. $\int_{\tilde{E}} f(x) d \mu_{n}(x) \rightarrow \int_{\tilde{E}} f(x) d \mu(x)$ for any $f \in C_{K}(\tilde{E})$. Here $C_{K}(A)$ is a set of continuous compact supported functions on $A$.

The next theorem is proved similarly to [4] Theorem 2.21.

TheOREM 2.11. $X$ is $\mu$-symmetric, i.e,

$$
\int_{\tilde{E}} P_{t} f(x) g(x) d \mu(x)=\int_{\tilde{E}} f(x) P_{t} g(x) d \mu(x) \quad \text { for any } f, g \in C_{K}(\tilde{E}) .
$$

In the end of this section, we give relations of scaling factors and remark about the spectral dimensions of nested fractals .

LEMMA 2.12.

1) Let $H_{m}=\sum_{0 \leq r \leq T_{1}^{0}\left(X\left(m, x_{0}\right)\right)} 1_{\left\{X\left(m, x_{0}\right)(r)=x_{0}\right\}}$. (We omit $m$ when $m=1$.)

Then, $E\left(H_{m}\right)=\{E(H)\}^{m}$.

2) $E(H)=(1-c)^{-1}=\frac{t_{E}}{N}$, where $c=P^{0}\left(\inf \{i>0: X(1,0)(i)=0\}<T_{1}^{0}(X(1,0))\right)$.

ProOF:

1) is proved in the same way as [4] Lemma 2.2 (b).

2) By the definition of $H$,

$$
\begin{aligned}
E(H) \cdot(1-c) & =\sum_{n=1}^{\infty} n P(H=n)(1-c) \\
& =\sum_{n=1}^{\infty} n c^{n-1}(1-c)^{2}=1 .
\end{aligned}
$$

Thus, $E(H)=(1-c)^{-1}$.

Let $\Delta$ be the infinitesimal generator of the reflecting Brownian motion on $E$. Using the Dynkin formula, for $f, g \in \mathcal{D}(\Delta)$, we have

$$
-\int_{E} \Delta f(x) g(x) d \mu(x)=\lim _{n \rightarrow \infty}\left(\frac{t_{E}}{N}\right)^{n} \sum_{x \in F^{(n)}} E(f(x)-f(X(n, x)(1))) g(x) \rho(x)\left(\sharp F^{(0)}\right)^{-1} .
$$

If we compare this with (4.5) of Kusuoka [8], we have $(1-c)^{-1}=\frac{t_{E}}{N}$.

Proposition 2.13. (Lindstrøm [9])

Let $\rho(x)$ be defined by $\rho(x)=\sharp\{\lambda \mid \lambda$ is a eigenvalue of $-\Delta, \lambda \leq x\}$.

If we let $d_{s}=\frac{2 \log N}{\log t_{E}}$, we have

$0<\liminf _{x \rightarrow \infty} \rho(x) / x^{\frac{d_{s}}{2}} \leq \limsup _{x \rightarrow \infty} \rho(x) / x^{\frac{d_{s}}{2}}<+\infty$.

We call this $d_{s}$ the spectral dimension of the nested fractal. 


\section{$\S 3 \quad$ Estimates of the hitting times.}

In this section, we will have the exponential estimates of the hitting time $W$.

LEMma 3.1. Let $\mathcal{F}=\left\{f\left(s_{1}, \cdots, s_{r}\right): f\right.$ is analytic in $\{\|x\| \leq 1\}$ and the Taylor expansion is $f=\sum a_{i_{1}, \cdots, i_{r}} s_{1}^{i_{1}} \cdots s_{r}^{i_{r}}$ where $\quad a_{i_{1}, \cdots, i_{r}} \geq 0$. Further, $\left.f(1, \cdots, 1) \leq 1\right\}$.

Then, there exist $S^{i} \subset\left\{\left(i_{1}, \cdots, i_{r}\right): i_{1}, \cdots, i_{r} \in \mathbb{Z}_{+}\right\}, \sharp S^{i}<\infty$ and

$$
g_{i_{1}, \cdots, i_{r}}^{i} \in \mathcal{F}, g_{i_{1}, \cdots, i_{r}}^{i}(0, \cdots, 0)>0 \text { such that }
$$

$$
f^{i}\left(s_{1}, \cdots, s_{r}\right)=\sum_{\left(i_{1}, \cdots, i_{r}\right) \in S^{i}} s_{1}^{i_{1}} \cdots s_{r}^{i_{r}} g_{i_{1}, \cdots, i_{r}}^{i_{1}}\left(s_{1}, \cdots, s_{r}\right)
$$

Proof: Fix $x \in F^{(0)}$ and consider all the 1 -walks $x_{0}, \cdots, x_{n}(n \in \mathbb{N})$ which satisfy $x_{0}=x, x_{n}=N^{i}(x), x_{1}, \cdots, x_{n-1} \in F^{(1)}-F^{(0)}$ and which do not pass the same points twice. Let $\left(i_{1}, \cdots, i_{r}\right)$ be the number of $\left\langle i_{j}>\right.$-type paths $(1 \leq j \leq r)$ for these paths. Then $S^{i}$ is a set of these $\left(i_{1}, \cdots, i_{r}\right)$.

REMARK: In fact, such a partial factorization holds for all $f^{i} \in \mathcal{F}$ in general.

In the following, we pick the above $S^{i}$ and fix it. (In fact, there is a smallest $S^{i}$ which satisfies Lemma 3.1 , but I do not mention it here.)

Proposition 3.2. If we have $0<\gamma<1$, and $\mathbf{x}=\left(x_{1}, \cdots, x_{r}\right)>0$ which satisfy

$$
(G(\mathbf{x}))_{i} \equiv \min _{\left(i_{1}, \cdots, i_{r}\right) \in S^{i}}\left\{\sum_{j=1}^{r} i_{j} x_{j}\right\}=t_{E}^{\gamma} x_{i} \quad 1 \leq i \leq r
$$

then there exist positive constants $c_{1 i} \sim c_{3 i}(1 \leq i \leq r)$ such that

$$
\exp \left(-c_{1 i} s^{1 / d_{J}}\right) \leq \phi_{i}(s) \leq c_{2 i} \exp \left(-c_{3 i} s^{1 / d_{J}}\right) \quad(1 \leq i \leq r)
$$

where $d_{J}=\gamma^{-1}$.

REMARK: This proposition, which is the reduction of the problem to some eigenvalue problem, is suggested by Kusuoka.

ProOF:

1) Proof of the upjer estimates: Take sufficiently small $M \in(0,1)$ such that $\sum_{\left(i_{1}, \cdots, i_{r}\right) \in S^{i}} M^{i_{1}+\cdots+i_{r}} \leq M$. (We can take such $M$ because constant term and linear terms are zero in the Taylor expansion of $f^{i}$.)

Next, take sufficiently small $\delta>0$ such that

$$
\phi_{i}(s) \leq M \exp \left(-\delta x_{i} s^{\gamma}\right) \quad \text { for } s \in\left[1, t_{E}\right]
$$


Then,

$$
\begin{aligned}
\phi_{i}\left(t_{E} s\right) & =f^{i}\left(\phi_{1}(s), \cdots, \phi_{r}(s)\right) \\
& =\sum_{S^{i}} \phi_{1}^{i_{1}} \cdots \phi_{r}^{i_{r}} g_{i_{1}, \cdots, i_{r}}^{i}\left(\phi_{1}(s), \cdots, \phi_{r}(s)\right) \\
& \leq \sum_{S^{i}} M^{i_{1}+\cdots+i_{r}} \exp \left(-\delta\left(\sum_{j} i_{j} x_{j}\right) s^{\gamma}\right) \\
& \leq M \exp \left(-\delta \min _{S^{i}}\left(\sum_{j} i_{j} x_{j}\right) s^{\gamma}\right) \\
& =M \exp \left(-\delta t_{E}^{\gamma} x_{i} s^{\gamma}\right) \\
& =M \exp \left(-\delta x_{i}\left(t_{E} s\right)^{\gamma}\right) .
\end{aligned}
$$

Thus, (3.3) holds for $s \in\left[t_{E}, t_{E}^{2}\right]$, too. Inductively, we know (3.3) holds for $s \in[1, \infty)$. As $\phi_{i}(s) \leq 1$, retaking $M$ sufficiently large, we have the upper estimates.

2) Proof of the lower estimates: Let $\left(i_{1}^{0}, \cdots, i_{r}^{0}\right) \in S^{i}$ be the one which attains the minimum in $(G(\mathbf{x}))_{i}$. Take sufficiently large $M \in[1, \infty)$ such that

$g_{i_{1}^{0}, \cdots, i_{r}^{0}}^{i}(0, \cdots, 0) M^{i_{1}^{0}+\cdots+i_{r}^{0}} \geq M$. Next, for fixed $a>0$, take sufficiently large $L_{a}>0$ such that

$$
\phi_{i}(s) \geq M \exp \left(-L_{a} x_{i} s^{\gamma}\right) \quad \text { for } s \in\left[a, a t_{E}\right]
$$

Then,

$$
\begin{aligned}
\phi_{i}\left(t_{E} s\right) & =f^{i}\left(\phi_{1}(s), \cdots, \phi_{r}(s)\right) \\
& =\sum_{S^{i}} \phi_{1}^{i_{1}} \cdots \phi_{r}^{i_{r}} g_{i_{1}, \cdots, i_{r}}^{i}\left(\phi_{1}(s), \cdots, \phi_{r}(s)\right) \\
& \geq \phi_{1}^{i_{1}^{0}}(s) \cdots \phi_{r}^{i_{r}^{0}}(s) g_{i_{1}^{0}, \cdots, i_{r}^{0}}^{i}(0, \cdots, 0) \\
& \geq g_{i_{1}^{0}, \cdots, i_{r}^{0}}^{i}(0, \cdots, 0) M^{i_{1}^{0}+\cdots+i_{r}^{0}} \exp \left(-L_{a}\left(\sum_{j} i_{j}^{0} x_{j}\right) s^{\gamma}\right) \\
& \geq M \exp \left(-L_{a} x_{i}\left(t_{E} s\right)^{\gamma}\right) .
\end{aligned}
$$

Thus, (3.4) holds for $s \in\left[a t_{E}, a t_{E}^{2}\right]$, too. Inductively, we know (3.4) holds for $s \in[a, \infty)$.

On the other hand, if we let $f_{b}(s)=\phi_{i}(s)-e^{-b s^{\gamma}}$, we easily see $f_{b}(s) \geq 0$ for $0 \leq s \leq c_{b}$ where $c_{b}>0$ increases when $b$ increases. From these facts, we obtain the lower estimates.

By now, our problem reduces to find $\gamma$ and $\mathbf{x}$ which satisfy (3.1). We will find it by searching the properties of $G(\mathbf{x})$.

Lemma 3.3. Let $B=\left\{\mathbf{x} \in \mathbb{P}^{r} \mid 0 \leq x_{1} \leq \cdots \leq x_{r}\right\}$. Then, $G(B) \subset B$.

Proof: Fix $p \in F^{(0)}, q \in N^{i}(p), q^{\prime} \in N^{i-1}(p)$. Let $U_{q q^{\prime}}$ be the reflection map which maps $q$ to $q^{\prime}$. Define $V=\left\{z \in \mathbb{R}^{D}:\left|z-q^{\prime}\right| \leq|z-q|\right\}$. Also we define a map $T: \mathbb{R}^{D} \rightarrow \mathbb{R}^{D}$ by 


$$
T z=\left\{\begin{array}{lr}
z & \text { if } z \in V \\
U_{q q^{\prime}} z & \text { otherwise }
\end{array}\right.
$$

For $\mathbf{x} \in B, \quad i \geq 2$, let $(G(\mathbf{x}))_{i}=a_{1} x_{1}+\cdots+a_{r} x_{r}, \quad\left(a_{1}, \cdots, a_{r}\right) \in S^{i}$

Then we know that there exists at least one 1 -walk from $p$ to $q$ which has $<k>$-type paths $a_{k}$ times $(1 \leq k \leq r)$. $\left(x_{0}, \cdots, x_{m}\right.$ is called a $n$-walk if $x_{i} \in F^{(n)}$ and $x_{i}, x_{i+1}$ join in the same $n$-complex.) Express the 1-walk by $x_{0}, x_{1}, \cdots, x_{m}$, where $x_{0}=p, x_{m}=q$ and $m=$ $\sum a_{i}$. If we let type $\left(x_{i}, x_{i+1}\right)$ be the type of the path $\overline{x_{i} x_{i+1}}$ (and let type $\left(x_{i}, x_{i+1}\right)=0$ if $\left.x_{i}=x_{i+1}\right)$, we know type $\left(T x_{i}, T x_{i+1}\right) \leq \operatorname{type}\left(x_{i}, x_{i+1}\right)$ because $\left|T x_{i}-T x_{i+1}\right| \leq\left|x_{i}-x_{i+1}\right|$. Denote $a_{j}^{\prime}=\sharp\left\{\left(x_{i}, x_{i+1}\right): \operatorname{type}\left(T x_{i}, T x_{i+1}\right)=j\right\}, 0 \leq j \leq m-1$. Then we have

$(G(\mathbf{x}))_{i-1} \leq \sum a_{i}^{\prime} x_{i} \leq \sum a_{i} x_{i}=(G(\mathbf{x}))_{i}$ because $x \in B$

Proposition 3.4 .

Let $K=\{A: A$ is a $r \times r$-matrix such that for all the $l$, (l-th low of $\left.A) \in S^{l}.\right\}$, and $\lambda=\min _{A \in K}\{$ largest eigenvalue of $A\}$. Then, there exists $\mathbf{x}>0$ such that $G(\mathbf{x})=\lambda \mathbf{x}$.

REMARK: The original proof of this by the author was not so elegant. The following is a shorter proof by Kusuoka.

Proof: If $\mathbf{x} \in B$, then $(G(\mathbf{x}))_{1} \geq x_{1}$ and $(G(\mathbf{x}))_{i} \leq c_{i} x_{1}$ for some $c_{i}>0(1 \leq i \leq r)$ because $\left(c_{i}, 0, \cdots, 0\right) \in S^{i}$ from Proposition 1.7 (3). Thus, if $x \in B$ and $x_{1}>0$, we know $\frac{(G(\mathbf{x}))_{1}}{\sum_{i}(G(\mathbf{x}))_{i}} \geq \frac{x_{1}}{\sum c_{i} x_{1}}=\frac{1}{\sum c_{i}} \equiv \epsilon$.

Let $B_{\epsilon}=\left\{x \in B: \sum x_{i}=1, x_{1} \geq \epsilon\right\}$ and $\tilde{G}(\mathbf{x})=\frac{1}{\sum(G(\mathbf{x}))_{i}} G(\mathbf{x})$ for $\mathbf{x} \in B_{\epsilon}$.

Then, by definition, $(\tilde{G}(\mathbf{x}))_{1} \geq \epsilon$. Combining this with Lemma 3.3, we know $\tilde{G}\left(B_{\epsilon}\right) \subset B_{\epsilon}$. Thus, by the fixed point theorem, there exists $\mathbf{x} \in B_{\epsilon}$ such that $\tilde{G}(\mathbf{x})=\mathbf{x}$. If we define $\lambda^{\prime}=\sum(G(\mathbf{x}))_{i}$, we have $G(\mathbf{x})=\lambda^{\prime} \mathbf{x}$. By the Frobenius theorem, it is easy to deduce $\lambda=\lambda^{\prime}$.

Definition 3.5. For $x, y \in F^{(n)} \cap E$, let

$d_{n}(x, y)=\{$ The shortest length of $n$-walk which moves from $x$ to $y$. $\}$.

If there exists $\rho>0$ such that

$0<\min _{x, y \in F^{(0)} \cap E, x \neq y} \liminf _{n \rightarrow \infty} \frac{d_{n}(x, y)}{\rho^{n}}$

$\leq \max _{x, y \in F^{(0)} \cap E, x \neq y} \lim \sup _{n \rightarrow \infty} \frac{d_{n}(x, y)}{\rho^{n}}<\infty$,

then we call $\rho$ : "growth rate of the length of the shortest path".

Proposition 3.6. The above $\rho$ exists in nested fractals. In fact, $\rho=\frac{\lambda}{\alpha}$.

ProOF: Let $\mathbf{y}=\left(\begin{array}{c}l_{1} \\ \vdots \\ l_{r}\end{array}\right)$ and take $a, b \in F^{(0)} \cap E$ such that $|a-b|=l_{j}$. Then it is easy to prove $d_{i}(a, b)=\left(\mathbf{e}_{j}, G^{\circ i}\left(\frac{\mathbf{y}}{\alpha^{i}}\right)\right)$, where $G^{\circ i}$ is the $i$-th composition of $G$ and $($,$) is a inner$ product. For $\mathbf{x}$ in Proposition 3.4, take $r>1$ such that $\frac{1}{r} \mathbf{x} \leq \mathbf{y} \leq r \mathbf{x}$. Then 
$\left(\mathbf{e}_{j}, G^{\circ i}\left(\frac{\mathbf{x}}{r \alpha^{i}}\right)\right) \leq\left(\mathbf{e}_{j}, G^{\circ i}\left(\frac{\mathbf{y}}{\alpha^{i}}\right)\right) \leq\left(\mathbf{e}_{j}, G^{\circ i}\left(\frac{r \mathbf{x}}{\alpha^{i}}\right)\right)$.

Thus, we have $\frac{1}{r} x_{j}\left(\frac{\lambda}{\alpha}\right)^{i} \leq d_{i}(a, b) \leq r x_{j}\left(\frac{\lambda}{\alpha}\right)^{i}$.

As $x_{j}>0$ we know that $\rho$ exists and $\rho=\frac{\lambda}{\alpha}$.

REMARK: We have $1 \leq \rho<t_{E} / \alpha$. The first inequality is trivial and the second comes from $\lambda \leq N$ and $t_{E}=N E(H)$ (c.f. Lemma 2.12).

From the above remark, if we define $\gamma=\frac{\log \alpha \rho}{\log t_{E}}$, then we know $0<\gamma<1$. Thus we have $\gamma$ and $\mathbf{x}$ which satisfy $(3.1)$.

Let $\phi(s)=E\left(e^{-s W}\right)=\sum_{i=1}^{r} m_{i} p_{i} \phi_{i}(s)$. Then, the next theorem is proved in the same way as Barlow-Perkins [4] Corollary 3.3 and Theorem 4.3.

THEOREM 3.7. There exist positive constants $c_{3.1} \sim c_{3.9}$ such that

$$
\begin{gathered}
\exp \left(-c_{3.1} s^{1 / d_{J}}\right) \leq \phi(s) \leq c_{3.2} \exp \left(-c_{3.3} s^{1 / d_{J}}\right) \\
c_{3.4} \exp \left(-c_{3.5} s^{-1 /\left(d_{J}-1\right)}\right) \leq P(W \leq s) \leq c_{3.6} \exp \left(-c_{3.7} s^{-1 /\left(d_{J}-1\right)}\right), \\
P^{x}\left(\sup _{s \leq t}\left|X_{s}-X_{0}\right| \geq \delta\right) \leq c_{3.8} \exp \left(-c_{3.9}\left(\delta^{d_{w}} t^{-1}\right)^{1 /\left(d_{J}-1\right)}\right),
\end{gathered}
$$

where $d_{J}=\frac{\log t_{E}}{\log \alpha \rho}$

\section{$\S 4$ Estimates of the Resolvent Densities.}

In this section, we will estimate the resolvent densities. As the proofs are essentially the same as [3],[4], we omit them.

Let $D_{m}(x)=E_{m}(x) \cap\left\{C: C\right.$ is a $m$-complex which is connected to $E_{m}(x)$. $\}$. Here $E_{m}(x)$ is the $m$-complex which contains $x$. (If there are more than one $m$-complexes which contain $x$, then choose one arbitrarily and fix it.)

Also, let $R_{\lambda}$ be an independent exponential random variable with mean $\lambda^{-1}$.

In the following, we fix $n \in \mathbb{Z}$, and let $x \in \tilde{E}, A=\operatorname{int} D_{n}(x)$.

We denote

$$
\begin{aligned}
R^{n}(A) & =\inf \left\{t \geq 0: X_{t}^{(n)} \in A^{c}\right\} \\
R(A) & =\inf \left\{t \geq 0: X_{t} \in A^{c}\right\} .
\end{aligned}
$$

Also, we define

$$
\begin{aligned}
U_{A}^{\lambda} f(x) & =E^{x}\left(\int_{0}^{R(A)} e^{-\lambda s} f\left(X_{s}\right) d s\right) \\
& =E^{x}\left(\int_{0}^{R_{\lambda} \wedge R(A)} f\left(X_{s}\right) d s\right) \quad \text { for } \lambda \geq 0, f \in b \mathcal{B}(\tilde{E})
\end{aligned}
$$

$(b \mathcal{B}(A)$ is a set of bounded Borel measurable functions on $A)$, 


$$
U^{\lambda} f(x)=E^{x}\left(\int_{0}^{\infty} e^{-\lambda s} f\left(X_{s}\right) d s\right) .
$$

Then, in the same way as Barlow-Perkins [4] $\S 5$, we know the existence of symmetric continuous resolvent densities $u_{A}^{\lambda}(x, y), u^{\lambda}(x, y)$ which satisfy

$$
\begin{aligned}
& U_{A}^{\lambda} f(x)=\int u_{A}^{\lambda}(x, y) f(y) d \mu(y), \\
& U^{\lambda} f(x)=\int u^{\lambda}(x, y) f(y) d \mu(y) .
\end{aligned}
$$

Now the following theorem can be proved in the same way as Barlow-Bass [3].

THEOREM 4.1.

(a) For $\lambda \geq 0, x, x^{\prime}, y \in \tilde{E}$, and $f \in \mathbb{L}^{1}(\tilde{E}, d \mu) \cap \mathbb{L}^{\infty}(\tilde{E}, d \mu)$, we have

$$
\begin{aligned}
\left|u_{A}^{\lambda}(x, y)-u_{A}^{\lambda}\left(x^{\prime}, y\right)\right| & \leq c_{4.1}\left|x-x^{\prime}\right|^{d_{w}-d_{f}}, \\
\left|U_{A}^{\lambda} f(x)-U_{A}^{\lambda} f\left(x^{\prime}\right)\right| & \leq c_{4.1}\left|x-x^{\prime}\right|^{d_{w}-d_{f}}\left\|f 1_{A}\right\|_{1} .
\end{aligned}
$$

(b) For $\lambda>0, x, x^{\prime} \in \tilde{E}, f \in \mathbb{L}^{\infty}(\tilde{E}, d \mu)$,

$$
\begin{gathered}
\left|U_{A}^{\lambda} f(x)-U_{A}^{\lambda} f\left(x^{\prime}\right)\right| \leq c_{4.2} \lambda^{-\frac{1}{2} d_{s}}\left|x-x^{\prime}\right|^{d_{w}-d_{f}}\|f\|_{\infty}, \\
c_{4.3}^{-1} \lambda^{\frac{1}{2} d_{s}-1} \leq u^{\lambda}(x, x) \leq c_{4.3} \lambda^{\frac{1}{2} d_{s}-1} .
\end{gathered}
$$

(c) (a),(b) hold for $u^{\lambda}, U^{\lambda}$ if $\lambda>0$.

\section{$\S 5$ Eigenvalue expansions and estimates of the transition densities.}

In this section, we will have the estimates of transition densities. We will follow the way of Barlow-Bass [3]. We omit most of proofs because they are the same as Barlow-Bass [3].

We fix $x_{0} \in \tilde{E}$ and $r \in \mathbb{Z}$. By the Mercer expansion theorem, we have a nonincreasing sequence of reals $\gamma_{j}>0$ and an orthonormal sequence $\varphi_{j}$ in $\mathbb{L}^{2}\left(D_{r}\left(x_{0}\right), d \mu\right)$ such that

$$
\begin{gathered}
u_{D_{r}\left(x_{0}\right)}^{\lambda}(x, y)=\sum_{j=1}^{\infty} \gamma_{j} \varphi_{j}(x) \varphi_{j}(y), \\
U_{D_{r}\left(x_{0}\right)}^{\lambda} f(x)=\sum_{j=1}^{\infty} \gamma_{j}\left(f, \varphi_{j}\right) \varphi_{j}(x), \quad f \in \mathbb{L}^{2}\left(D_{r}\left(x_{0}\right), d \mu\right) .
\end{gathered}
$$

The sum in (5.1) and (5.2) converges uniformly as well as in $\mathbb{L}^{2}$. Set $\lambda_{j}=\gamma_{j}^{-1}-\lambda$. Define 


$$
p_{D_{r}\left(x_{0}\right)}(t, x, y)=\sum_{j=1}^{\infty} e^{-\lambda_{j} t} \varphi_{j}(x) \varphi_{j}(y), \quad x, y \in D_{r}\left(x_{0}\right)
$$

Then, we can prove that (5.3) converges absolutely and uniformly on $D_{r}\left(x_{0}\right)$, $p_{D_{r}\left(x_{0}\right)}(t, x, y)$ is a version of the transition densities for $\left(P^{x}, X_{t}\right)$ killed on exiting $D_{r}\left(x_{0}\right)$, and is jointly continuous in $(t, x, y)$ on $(0, \infty) \times \tilde{E} \times \tilde{E}$. Clearly $p_{D_{r}\left(x_{0}\right)}(t, x, y)$ increases as $r$ decreases. Let us define

$$
p(t, x, y)=\lim _{r \rightarrow-\infty} p_{D_{r}\left(x_{0}\right)}(t, x, y)
$$

Then we have

THEOREM 5.1.

(a) $p(t, x, y)$ is a version of the transition density of $\left(P^{x}, X_{t}\right)$ with respect to $\mu$.

(b) $p(t, x, y)$ is symmetric in $x$ and $y$.

(c) $p(t, x, y) \leq c_{5.1} t^{-\frac{d_{s}}{2}}$.

(d) $p(t, x, y)$ is jointly continuous in $(t, x, y)$ and $\left|p(t, x, y)-p\left(t, x^{\prime}, y\right)\right| \leq c_{5.2} t^{-1}\left|x-x^{\prime}\right|^{d_{w}-d_{f}}$.

(e) $p(t, x, y)$ is $C^{\infty}$ in $t$ and $\partial^{k} p(t, x, y) / \partial t^{k}$ is Hölder continuous of order $d_{w}-d_{f}$ in each space variable.

THEOREM 5.2. (Upper bounds ) There exist positive constants $c_{5.3}, c_{5.4}$ such that

$$
p(t, x, y) \leq c_{5.3} t^{-d_{s} / 2} \exp \left(-c_{5.4}\left(|x-y|^{d_{w}} / t\right)^{1 /\left(d_{J}-1\right)}\right), \quad x, y \in \tilde{E}
$$

LEMMA 5.3.

1) There exists $c_{5.5}>0$ such that

$$
p(t, x, x) \geq c_{5.5} t^{-d_{s} / 2}
$$

2) There exist positive constants $c_{5.6}, c_{5.7}$ such that

$$
p(t, x, y) \geq c_{5.6} t^{-d_{s} / 2} \quad \text { for }|x-y| \leq c_{5.7} t^{1 / d_{w}} .
$$

Lemma 5.4. There exists $c_{5.8}>0$ which satisfies the following for all the $x, y \in \tilde{E}, m \in \mathbb{Z}$ and $k \in \mathbb{N}$ : 
"If $|x-y|<\alpha^{-m}$, then there exist $x_{0}, x_{1}, \cdots, x_{n}\left(n \leq c_{5.8}(\alpha \rho)^{k}\right)$ such that $x_{0}=x, x_{n}=$ $y, x_{1}, \cdots, x_{n-1} \in F^{(m+k)}$ and $x_{i}, x_{i+1}$ join in the same $(m+k)$-complex for $0 \leq i \leq n-1$." ProOF: First, we prove $\lim \sup _{n \rightarrow \infty} \max _{x \in F^{(n)} \cap E} \max _{y \in F^{(0)} \cap E} \frac{\tilde{d}_{n}(x, y)}{(\alpha \rho)^{n}}<\infty$, where $\tilde{d}_{n}(x, y)=\{$ Number of the step for the shortest $n$-walk leading $x$ to $y$. $\}$.

Let $A_{i}=\max _{x, y \in F^{(0)} \cap E} \tilde{d}_{i}(x, y)$ and $q=\max _{x \in F^{(1)} \cap E} \max _{y \in F^{(0)} \cap E} \tilde{d}_{1}(x, y)$. Then, we easily see $\max _{x \in F^{(n)} \cap E} \max _{y \in F^{(0)} \cap E} \tilde{d}_{n}(x, y) \leq q \sum_{k=1}^{n-1} A_{k}+q$.

By Proposition 3.6, we know that there exists $c>1$ such that $A_{n} \leq c(\alpha \rho)^{n}$. Thus, we have

$$
\begin{aligned}
\max _{x \in F^{(n)} \cap E} \max _{y \in F^{(0)} \cap E} \frac{\tilde{d}_{n}(x, y)}{(\alpha \rho)^{n}} & \leq q \sum_{k=1}^{n-1} \frac{A_{k}}{(\alpha \rho)^{n}}+\frac{q}{(\alpha \rho)^{n}} \\
& \leq q c \sum_{k=1}^{n-1} \frac{1}{(\alpha \rho)^{k}}+\frac{q}{(\alpha \rho)^{n}} \\
& \leq \frac{q c}{\alpha \rho-1} .
\end{aligned}
$$

Now, if $|x-y|<\alpha^{-m}$, then by Assumption 2.2, we have $x_{0}, x_{1}, \cdots, x_{l}$ such that $x_{0}=$ $x, x_{l}=y, x_{1}, \cdots, x_{l-1} \in F^{(m)}$ and $x_{i}, x_{i+1}$ join in the same $m$-complex for $0 \leq i \leq l-1$. Take $x_{0}^{\prime} \in F^{(m+k)}$ which joins in the same $(m+k)$-complex as $x_{0}$ and $x^{\prime}{ }_{l}$ in the same way. Then, by the fact we proved above, we know that we can make a sequence of $F^{(m+k)}$ points which connects $x_{i}$ and $x_{i+1}$ for $0 \leq i \leq l-1$ with at most $c(\alpha \rho)^{k}$ points for some large $c$.

TheOREM 5.5. (Lower bounds ) There exist positive constants $c_{5.9}, c_{5.10}$ such that

$$
p(t, x, y) \geq c_{5.9} t^{-d_{s} / 2} \exp \left(-c_{5.10}\left(|x-y|^{d_{w}} / t\right)^{1 /\left(d_{J}-1\right)}\right), \quad x, y \in \tilde{E} .
$$

Proof: The idea of the proof is just the same as Barlow-Bass [3], but we need some modifications.

Let $D=|x-y|$. By Lemma 5.3, the theorem is already proved if $D \leq c_{5.7} t^{1 / d_{w}}$. Thus we assume $D \geq c_{5.7} t^{1 / d_{w}}$. We may find $c_{5.11}$ depending only on $\alpha, c_{5.7}$ and $d_{w}$ which satisfies the following:

"If we take $k$ such that

$\left(t_{E} /(\alpha \rho)\right)^{k}>c_{5.11} t^{-1} D^{d_{w}} \geq\left(t_{E} /(\alpha \rho)\right)^{k-1}$, then $2 D / \alpha^{k+1} \leq c_{5.7}\left(t /(\alpha \rho)^{k}\right)^{1 / d_{w}} . "$

Now take $m$ such that $\alpha^{-m-1} \leq D<\alpha^{-m}$. By Lemma 5.4, we can pick the sequence $x_{0}, \cdots, x_{n}\left(n \leq c_{5.8}(\alpha \rho)^{k}\right)$ such that $x_{0}=x, x_{n}=y, x_{1}, \cdots, x_{n-1} \in F^{(m+k)}$ and $x_{i}, x_{i+1}$ join in the same $(m+k)$-complex for $0 \leq i \leq n-1$. Let $\epsilon=D /\left(2 \alpha^{k+1}\right)$ and $B_{i}=$ $B\left(x_{i}, \epsilon\right) \cap \tilde{E}$. Note that if $z \in B_{i-1}, z^{\prime} \in B_{i}$,

$$
\left|z-z^{\prime}\right| \leq 2 \epsilon+D / \alpha^{k+1}=2 D / \alpha^{k+1} \leq c_{5.7}\left(t /(\alpha \rho)^{k}\right)^{1 / d_{w}},
$$


so that $p\left(t /(\alpha \rho)^{k}, z_{i-1}, z_{i}\right) \geq c_{5.6}\left(t /(\alpha \rho)^{k}\right)^{-d_{s} / 2}$. Then,

$$
\begin{aligned}
p(t, x, y) & \geq \int_{B_{1}} \cdots \int_{B_{n-1}} p\left(t /(\alpha \rho)^{k}, x, y_{1}\right) \cdots p\left(t /(\alpha \rho)^{k}, y_{n-1}, y\right) d \mu\left(y_{1}\right) \cdots d \mu\left(y_{n-1}\right) \\
& \geq\left(\Pi_{i=1}^{n-1} \mu\left(B_{i}\right)\right) c_{5.6}^{n}\left(t /(\alpha \rho)^{k}\right)^{-d_{s} n / 2} \\
& \geq c_{5.12}^{n}\left(D /\left(2 \alpha^{k+1}\right)\right)^{d_{f}(n-1)}\left(t /(\alpha \rho)^{k}\right)^{-d_{s} n / 2} .
\end{aligned}
$$

Since $d_{s} / 2=d_{f} / d_{w}$ and by our choice of $k,\left(D /\left(2 \alpha^{k+1}\right)\right) /\left(t /(\alpha \rho)^{k}\right)^{1 / d_{w}}$ is bounded above and below by positive constants which are independent of $D$ and $t$. Thus, we have

$$
\begin{aligned}
p(t, x, y) & \geq c_{5.13}^{n} c_{5.14}\left(t /(\alpha \rho)^{k}\right)^{-d_{s} / 2} \\
& \geq c_{5.13}^{n} c_{5.15} t^{-d_{s} / 2} \\
& \geq c_{5.15} t^{-d_{s} / 2} \exp \left(-c_{5.8}(\alpha \rho)^{k} \log c_{5.13}^{-1}\right) .
\end{aligned}
$$

Substituting our choice in the last term completes the proof.

Combining Theorem 5.1, Theorem 5.2 and Theorem 5.5, we obtain the estimates of the transition densities.

\section{$\S 6$ Some remarks}

As Barlow-Bass [3] has written in Section 8, various estimates holds for the Brownian motion on nested fractals and proofs are essentially the same. Here we will introduce properties about sample path, local time and domain of the generator. The readers can prove them in the same way as [3],[4].

THEOREM 6.1.

a) There are positive constants $c_{6.1}, c_{6.2}$ such that

$$
c_{6.1} t^{p / d_{w}} \leq E^{x}\left|X_{t}-x\right|^{p} \leq c_{6.2} t^{p / d_{w}}
$$

b) $X$ has a modulus of continuity given by

$$
c_{6.3} \leq \lim _{\delta \rightarrow 0} \sup _{0 \leq s \leq t \leq T,|s-t| \leq \delta} \frac{\left|X_{t}-X_{s}\right|}{|s-t|^{1 / d_{w}}(\log 1 /|s-t|)^{\left(d_{J}-1\right) / d_{w}}} \leq c_{6.4} .
$$

c) If $T_{x}^{+}=\inf \left\{t>0: X_{t}=x\right\}$, then $P^{x}\left(T_{x}^{+}=0\right)=1$, so that for all $x \in \tilde{E}, x$ is regular for $\{x\}$.

d) For each $x, y \in \tilde{E}, P^{x}\left(T_{y}<\infty\right)=1$.

e) $\left\{t: X_{t}=x\right\}$ is $P^{y}$-a.s. perfect and unbounded, so that $X$ is point recurrent.

REMARK. It is easy to obtain $t_{E} \geq \alpha^{2}$. Thus we have $d_{w} \geq 2$. 
THEOREM 6.2. There exists a jointly continuous version $L_{t}^{x}$ of the local time of $X$ which satisfies the density of occupation formula:

$$
\int_{0}^{t} f\left(X_{s}\right) d s=\int_{\tilde{E}} f(y) L_{t}^{y} d \mu(y) \quad \text { for } f \in C_{K}(\tilde{E})
$$

and has modulus of continuity given by :

$$
\lim _{\delta \rightarrow 0} \sup _{0 \leq s \leq t,|s-t| \leq \delta} \frac{\left|L_{s}^{x}-L_{s}^{y}\right|}{\varphi(|x-y|)} \leq c_{6.5}\left(\sup _{z \in \tilde{E}} L_{t}^{z}\right)^{\frac{1}{2}}
$$

where $\varphi(u)=u^{\frac{1}{2}\left(d_{w}-d_{f}\right)}(\log 1 / u)^{\frac{1}{2}}$.

REMARK. Barlow suggested me that the above modulus of continuity holds on nested fractals.

TheOrem 6.3. Let $C_{0}(\tilde{E})$ be the set of continuous functions on $\tilde{E}$ vanishing at $\infty$. Let $(\Delta, \mathcal{D}(\Delta))$ be the infinitesimal generator of $\left\{P_{t}\right\}$. Then, we have the following .

a) $P_{t}: C_{0}(\tilde{E}) \rightarrow C_{o}(\tilde{E})$, and $\left\{P_{t}\right\}$ is a strong Feller semigroup on $C_{0}(\tilde{E})$.

b) Every function in $\mathcal{D}(\Delta)$ is Hölder continuous of order $d_{w}-d_{f}$.

c) For $y_{0} \in \tilde{E}$, the function $p\left(\cdot, \cdot, y_{0}\right)$ is a solution of the heat equation on $\tilde{E}$ :

$$
\frac{\partial}{\partial t} p\left(t, x, y_{0}\right)=\Delta_{x} p\left(t, x, y_{0}\right), \quad t>0, x \in \tilde{E}
$$




\section{$\S 7$ Examples}

In this section we give some examples of the nested fractals.

Example 7.1 (Sierpinski gasket)

$\alpha=2, N=\sharp F^{(0)}=3, r=1, p_{1}=1 / 2, t_{E}=5, \rho=1$.

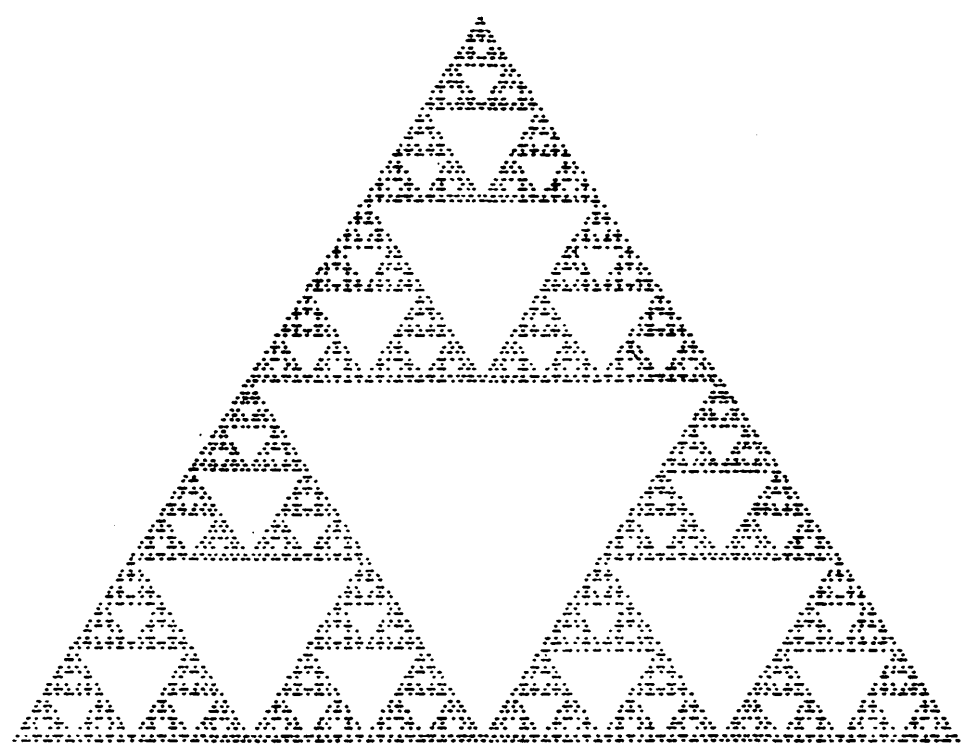

Example 7.2

$\alpha=5, N=12, \sharp F^{(0)}=3, r=1, p_{1}=1 / 2, \rho=6 / 5$.

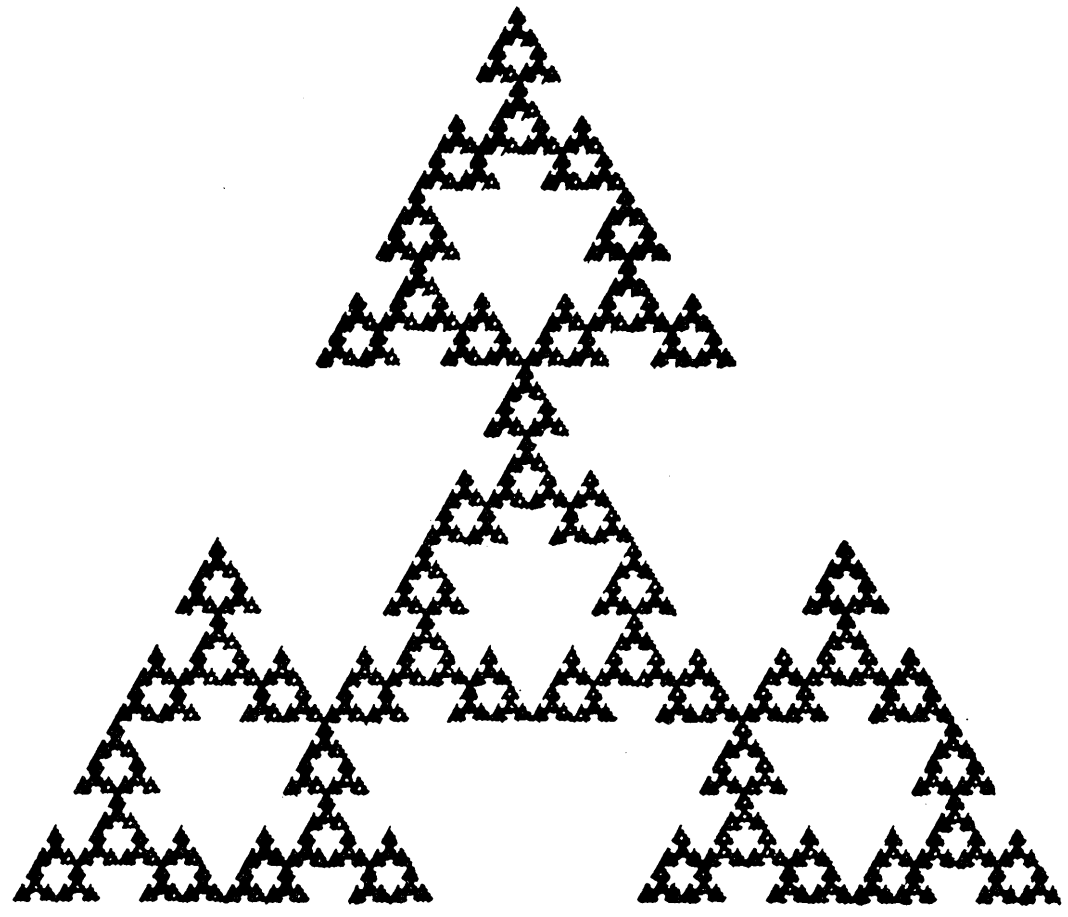


Example 7.3 (Pentakun)

$\alpha=(3+\sqrt{5}) / 2, N=\sharp F^{(0)}=5, r=2, p_{1}=(\sqrt{161}-7) / 16, p_{2}=(15-\sqrt{161}) / 16$, $t_{E}=(\sqrt{161}+9) / 2, \rho=(\sqrt{3}+1) / \alpha$.

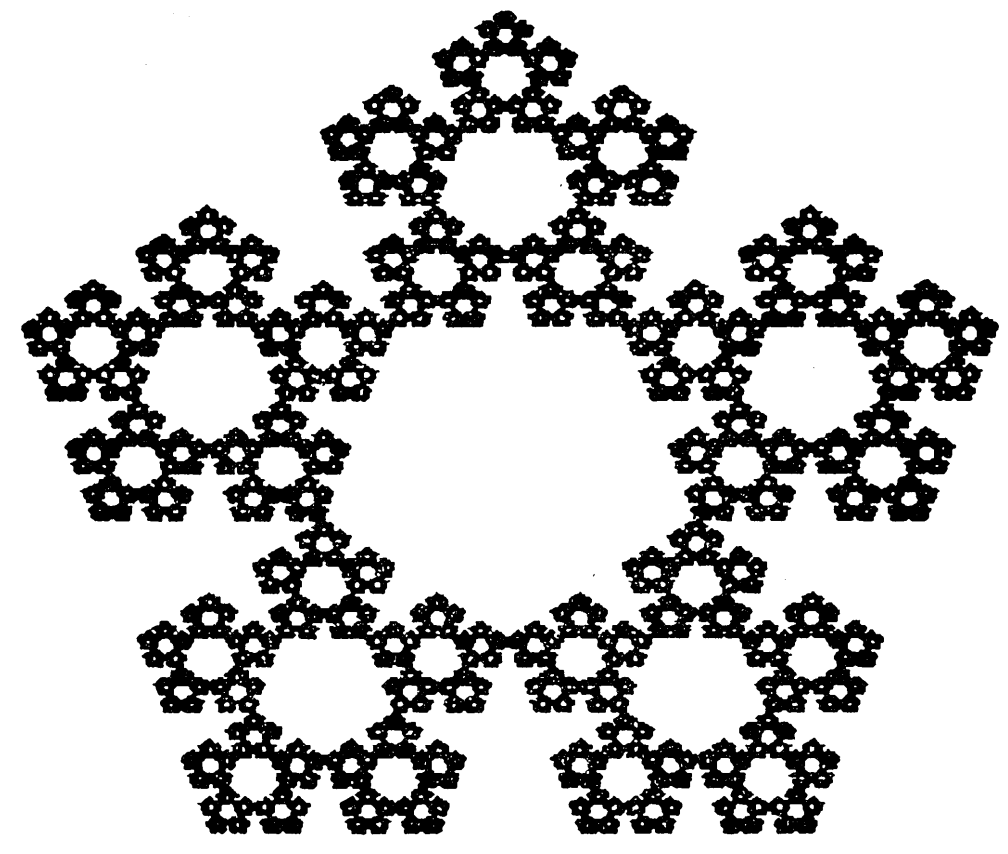

Example 7.4 (Lindstrøm's Snowflake)

$\alpha=3, N=7, \sharp F^{(0)}=6, r=3, \rho=1, p_{1} \fallingdotseq 0.29737$,

$p_{2} \fallingdotseq 0.14390, p_{3} \fallingdotseq 0.11746, t_{E} \fallingdotseq 12.89027$ (these are computed numerically, see [10]).

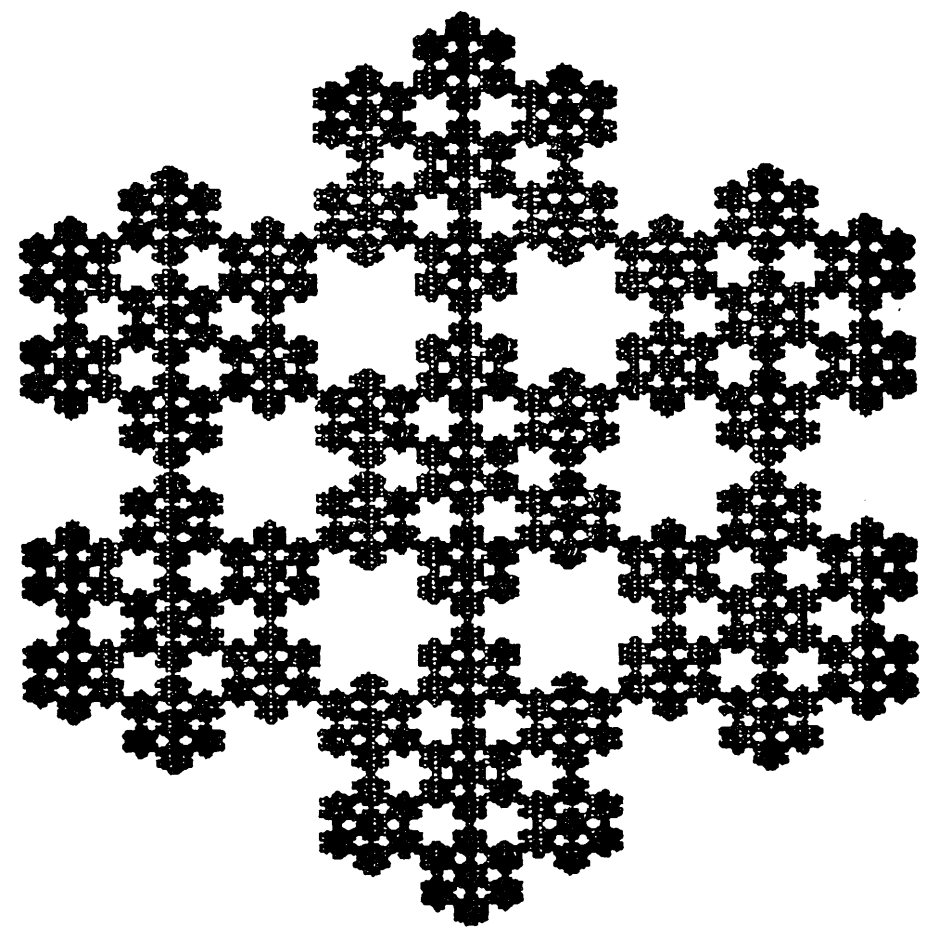




\section{REFERENCES.}

[1]. K.B.Athreya and P.E.Ney, Branching processes, Berlin/Heidelberg/New York, Springer (1972).

[2]. M.T.Barlow, Random walks, electrical resistance, and nested fractals, To Appear in "Asymptotic Problems in Probability Theory" (K.D.Elworthy \& N.Ikeda (eds.)), Pitman.

[3]. M.T.Barlow, and R.F.Bass, Transition Densities for Brownian Motion on the Sierpinski Carpet, To Appear in Prob. Th. Rel. Fields.

[4]. M.T.Barlow, and E.A.Perkins, Brownian motion on the Sierpinski gasket, Prob. Th. Rel. Fields 79 (1988), 543-623.

[5]. M.Fukushima, Dirichlet forms, diffusion processes and spectral dimensions for nested fractals, To appear in "Ideas and Methods in Mathematical Analysis, Stochastics, and Applications, In Memory of R. Høegh-Krohn, vol. 1" (Albeverio, Fenstad, Holden and Lindstrøm (eds.)), Cambridge Univ. Press.

[6]. Hutchinson, Fractals and Self-similarity, Indiana Univ. Math. J. 30 (1981).

[7]. T.Kumagai, Construction and some properties of a class of non-symmetric diffusion processes on the Sierpinski gasket, To Appear in "Asymptotic Problems in Probability Theory" (K.D.Elworthy \& N.Ikeda (eds.)), Pitman.

[8]. S.Kusuoka, Diffusion processes on nested fractals, Lecture Note at Nankai University (1989).

[9]. T.Lindstrøm, Brownian motion on nested fractals, Mem. Amer. Math. Soc. 420 (1990).

[10]. H.Yokoi, Denumerable Markov chains and nested fractals, Master thesis at Tohoku University (1991). 